PROCEEDINGS OF THE

AMERICAN MATHEMATICAL SOCIETY

Volume 131, Number 5, Pages 1539-1551

S 0002-9939(02)06740-0

Article electronically published on September 19, 2002

\title{
NONZERO FIXED POINTS OF POWER-BOUNDED LINEAR OPERATORS
}

EFE A. OK

(Communicated by Joseph A. Ball)

\begin{abstract}
This paper provides a variety of sufficient conditions for the existence of a nonzero fixed point of a power-bounded linear operator defined on a real Banach space. In the case of power-bounded positive operators on a Banach lattice, among the conditions we provide are not being strongly stable along with commuting with a compact operator or being quasicompact. These results apply directly to Markov operators. In the case of an arbitrary powerbounded operator on a Hilbert space, being uniformly asymptotically regular and not strongly stable guarantees the existence of a nonzero fixed point.
\end{abstract}

\section{INTRODUCTION}

This paper is concerned with the problem of determining whether or not a given power-bounded linear operator on a real Banach space has a nontrivial fixed point. A particularly interesting instant of the query is obtained in the case of nonexpansive linear operators (i.e. contractions). While nonexpansive maps are studied extensively within metric fixed point theory, this problem does not seem to have received much attention in the literature. It appears that most of the related work is done rather through ergodic theory in the special case of Markov operators. By contrast, our objective here is to treat the problem from an operator-theoretic viewpoint, and derive general nonzero fixed point theorems for contractions defined on real Banach spaces. By way of application, we then use these results to provide seemingly new sufficient conditions for the existence of invariant densities for Markov operators and homogeneous Markov chains.

After going through some preliminary nomenclature in Section 1, we focus in Section 2 on power-bounded positive operators defined on a real Banach lattice $X$. It is shown that any such operator $T$ that commutes with a compact operator $K$ has a nonzero fixed point if the norms of the $T$-iterations of at least one positive point in the image of $K$ does not converge to zero. The same conclusion is also obtained for positive and power-bounded Dunford-Pettis operators that commute with a weakly compact operator. In particular, if $T$ is power-compact, then $T$ must have a positive fixed point provided that it is not strongly stable on the positive cone. If $X$ is an AL-space, power-compactness can be replaced in this statement with

Received by the editors October 29, 2001 and, in revised form, December 17, 2001.

2000 Mathematics Subject Classification. Primary 47H09, 47H10; Secondary 47B07.

Key words and phrases. Fixed points, contractions, compact operators, Markov operators, strong stability, asymptotic regularity. 
quasicompactness. An immediate implication of these results is that if a Markov operator is quasicompact, or it commutes with a compact operator, then it has an invariant density (Section 3). Finally, in Section 4, we show that a uniformly asymptotically regular and power-bounded operator on a real Hilbert space has a nonzero fixed point if and only if it is not strongly stable. It is not known if this observation holds for non-uniformly asymptotically regular operators.

\section{Preliminaries}

We adopt the standard notation and terminology of the theory of linear operators. For any Banach space $X$, we let $B_{X}$ and $S_{X}$ stand for the closed unit ball and unit sphere in $X$, respectively. For any nonempty $S \subseteq X$, we denote by $\overline{\mathrm{co}}(S)$ the smallest closed convex set that contains $S$, and let $\operatorname{ext}(S)$ stand for the set of all extreme points of $S$. If $\left(e^{k}\right)$ is a Schauder basis for $X$, the associated $k$ th coordinate of a vector $x$ is denoted by $\left\langle x, e^{k}\right\rangle$, that is, $x=\sum\left\langle x, e^{k}\right\rangle e^{k}$ for every $x \in X$.

The Banach algebras of all linear and bounded linear operators on $X$ are denoted as $\mathcal{L}(X)$ and $\mathcal{B}(X)$, respectively. As usual, the adjoint, the spectrum, the point spectrum, the continuous spectrum, the residual spectrum, and the spectral radius of an operator $T \in \mathcal{B}(X)$ are denoted by $T^{*}, \sigma(T), \sigma_{p}(T), \sigma_{c}(T), \sigma_{r}(T)$, and $r(T)$, respectively. In turn, the set of compact and weakly compact operators on $X$ are denoted respectively as $\mathcal{K}(X)$ and $\mathcal{K}^{w}(X)$, both of which are closed ideals in $\mathcal{B}(X)$. Adopting the convention $T^{0}:=I$, we let $T^{n}$ stand for the $n$th iteration of $T$ for each nonnegative integer $n$. T is called algebraic if there exist an $n \in \mathbb{N}$ and $\alpha_{0}, \ldots, \alpha_{n} \in \mathbb{Z}$ such that $\sum_{i=1}^{n} \alpha_{i} T^{i}=0$. If $T^{n} \in \mathcal{K}(X)$ for some $n \in \mathbb{N}$, then $T$ is called power-compact, and if $\left\|T^{n}-K\right\|<1$ for some $(n, K) \in \mathbb{N} \times \mathcal{K}(X)$, then $T$ is called quasicompact. Weakly power-compact and weakly quasicompact operators are defined by replacing $\mathcal{K}(X)$ with $\mathcal{K}^{w}(X)$ in these definitions, respectively.

A linear operator $T$ on $X$ is called a contraction if $\|T\| \leq 1$. Obviously, due to its linearity, $T$ is a contraction if and only if it is nonexpansive, that is, $\|T x-T y\| \leq$ $\|x-y\|$ for all $x, y \in X$. One of the objectives of this paper is to investigate which sort of contractions have nonzero fixed points. The analysis will, however, be conducted in terms of a more general class of operators.

Definition. Let $X$ be a normed linear space and $T \in \mathcal{B}(X)$. We say that $T$ is power-bounded if

$$
\limsup _{n \rightarrow \infty}\left\|T^{n} x\right\|<\infty \quad \text { for all } x \in X .
$$

If $T \in \mathcal{B}(X)$ is similar to a contraction, that is, if there exists an invertible $U \in \mathcal{B}(X)$ with $\left\|U T U^{-1}\right\| \leq 1$, then $T$ is power-bounded, for in this case

$$
\left\|T^{n}\right\|=\left\|U^{-1} U T^{n} U^{-1} U\right\| \leq\left\|U^{-1}\right\|\left\|U T U^{-1}\right\|^{n}\|U\| \leq\left\|U^{-1}\right\|\|U\|
$$

for each $n \in \mathbb{N}$. The converse is not true; a power-bounded operator in $\mathcal{B}(X)$ need not be similar to a contraction (cf. Foguel [5]). Note also that, when $X$ is a Banach space, it follows from the uniform boundedness principle that $T \in \mathcal{B}(X)$ is powerbounded iff $\sup _{n \geq 1}\left\|T^{n}\right\|<\infty$. Thus, in this case, the power-boundedness property simply avoids the norms of the iterates of a bounded operator escape to infinity.

Obviously, a power-bounded operator $T \in \mathcal{B}(X)$ that has a nonzero fixed point cannot be strictly contractive (i.e. $\|T\|<1$ ), for the unique fixed point of a strictly contractive linear operator is 0 . The converse is clearly not true; even a contraction which is not strictly contractive (such as a rotation) need not have a nonzero 
fixed point. Thus one needs to assert stronger properties than "not being strictly contractive" for the power-bounded members of $\mathcal{B}(X)$ in order to guarantee the existence of a nonzero fixed point. Following the terminology of Kubrusly [10], the main property that we shall invoke here for this purpose is introduced next.

Definition. Let $X$ be a normed linear space and $T \in \mathcal{B}(X)$. We say that $T$ is strongly stable if

$$
\liminf _{n \rightarrow \infty}\left\|T^{n} x\right\|=0 \quad \text { for all } x \in X .
$$

"Not being strongly stable" is a local property that puts a positive lower bound on the norms of the $T$-iterations of a single point. It is thus satisfied by any isometry, or more generally, any $C_{1}$-contraction (cf. Nagy-Foias [15]). In contrast to powerboundedness, it can be thought of as avoiding the norms of the $T$-iterations of a given point escape to zero. So, at least locally, this property works counter to power boundedness. Consequently, in the presence of these two properties, one is likely to have a good control over the sequence of the norms of the local $T$-iterates. This intuition underlies much of what follows.

Finally, we introduce our lattice-theoretic terminology. Unless explicitly stated otherwise, by a Banach lattice we mean a real Banach lattice. For any Banach lattice $(X, \succsim)$, the positive cone $\{x \in X: x \succsim 0\}$ is denoted as $X_{+}$and $X_{++}:=$ $X_{+} \backslash\{0\}$. We recall that the antisymmetry of $\succsim$ implies that $X_{+} \cap-X_{+}=\{0\}$ and that an operator $T \in \mathcal{L}(X)$ is called positive if $T\left(X_{+}\right) \subseteq X_{+}$. We denote the set of all positive operators on $X$ as $\mathcal{L}_{+}(X)$ and note that $\mathcal{L}_{+}(X) \subseteq \mathcal{B}(X)$. An operator $T \in \mathcal{L}(X)$ is called positively weakly quasicompact if there exists an $(n, K) \in \mathbb{N} \times \mathcal{K}^{w}(X)$ such that both $K$ and $T^{n}-K$ are positive, and $\left\|T^{n}-K\right\|<1$. Obviously, every weakly power-compact operator in $\mathcal{L}_{+}(X)$ is positively weakly quasicompact.

\section{COMPaCtNess And POSITIVE FIXED POINTS OF POWER-BOUNDED OPERATORS}

Let $X$ be a Banach lattice. One obvious way of guaranteeing that a contraction $T \in \mathcal{L}_{+}(X)$ has a nonzero fixed point is to impose a restriction on the asymptotic behavior of the $T$-iterations of a given point in the space. For instance, if $\lim T^{n} x \in$ $X_{++}$for some $x \in X$, then $\lim T^{n} x$ is a nonzero fixed point of $T$. Or, less trivially, if $X$ is uniformly convex and there exists an $(x, y) \in X \times X_{++}$such that $T^{n}(x) \in$ $X_{+}+y$ for each $n$, then $T$ has a positive fixed point, because the Browder-Göhde fixed point theorem applies to the restriction of $T$ to $\overline{\operatorname{co}}\left\{T^{n} x: n \in \mathbb{N}\right\}$.

Of course imposing conditions directly on the sequence $\left(T^{n} x\right)$ is more demanding than working with the real sequence $\left(\left\|T^{n} x\right\|\right)$. For an isometry, for instance, the exact nature of the first sequence may be difficult to determine, while that of the latter is trivial. Motivated by this observation, the iterative conditions that are considered in this paper are imposed only on such real sequences. In particular, the main results of this section (Theorems 2.1 and 2.2) will show that "not being strongly stable" is enough to guarantee the existence of a nonzero fixed point for a positive contraction that satisfies relatively mild compactness postulates.

\subsection{The case of commutants of compact operators.}

Theorem 2.1.A. Let $X$ be a Banach lattice, and let $T \in \mathcal{L}_{+}(X)$ be a power bounded operator that commutes with a compact operator $K \in \mathcal{K}(X)$. Then $T$ has 
a nonzero fixed point in $X_{+}$provided that

$$
\liminf _{n \rightarrow \infty}\left\|T^{n} y\right\|>0 \quad \text { for some } y \in X_{+} \cap K(X) .
$$

Proof. Pick any $x \in K^{-1}(y)$ and observe that $x, y \neq 0$ by (3). Now, for any $z \in X$, let $\mathcal{O}_{T}(z)$ stand for the $T$-orbit of $z$. Since $T$ and $K$ commute, we have

$$
\mathcal{O}_{T}(y):=\left\{T^{n} y: n=0,1, \ldots\right\}=\left\{T^{n} K x: n=0,1, \ldots\right\}=K\left(\mathcal{O}_{T}(x)\right) .
$$

We let $A:=\operatorname{cl}\left(\mathcal{O}_{T}(y)\right)$. Since $T$ is power-bounded, $\left\{T^{n} x: n \in \mathbb{N}\right\}$ is a bounded set, so $K\left(\mathcal{O}_{T}(x)\right)$ is relatively compact. Thus $A$ is a nonempty compact set in $X$. Moreover,

$$
\begin{aligned}
T\left(\operatorname{cl}\left(\mathcal{O}_{T}(y)\right)\right) & \subseteq \operatorname{cl}\left(T\left(\mathcal{O}_{T}(y)\right)\right) \\
& =\operatorname{cl}\left(T K\left(\mathcal{O}_{T}(x)\right)\right) \\
& =\operatorname{cl}\left(K T\left(\mathcal{O}_{T}(x)\right)\right) \\
& \subseteq \operatorname{cl}\left(K\left(\mathcal{O}_{T}(x)\right)\right)
\end{aligned}
$$

so that $T(A) \subseteq A$. Next we define $S:=\overline{\mathrm{co}}(A)$ which is a convex set. Given that $A$ is compact, $S$ must be compact by Mazur's compactness theorem. Moreover, since $T(A) \subseteq A$, we have $T(\overline{\mathrm{co}}(A)) \subseteq \overline{\mathrm{co}}(T(A)) \subseteq \overline{\mathrm{co}}(A)$, that is, $T(S) \subseteq S$. Thus, $\left.T\right|_{S}$ is a continuous self-map on the nonempty convex and compact set $S$. By the Schauder-Tychonoff fixed point theorem, therefore, there exists a $z \in S$ such that $z=\left.T\right|_{S}(z)=T z$. To complete the proof, it is then enough to show that $S \subseteq X_{++}$.

Given that $T$ is positive and $y \in X_{+}$, we have $\mathcal{O}_{T}(y) \subseteq X_{+}$. Since the positive cone of any normed lattice is closed, $X_{+}$is a closed convex set in $X$, so $A \subseteq X_{+}$. This further implies that $S \subseteq X_{+}$. So all we need to show is that $0 \notin S$. Observe first that $0 \notin S \backslash \operatorname{ext}(S)$. Indeed, if $u$ and $v$ are two distinct vectors in $S$, then, since $S \subseteq X_{+}$, either $u \in X_{++}$or $v \in X_{++}$, and hence, given that $X_{+} \cap-X_{+}=\{0\}$, we have $\lambda u+(1-\lambda) v \in X_{++}$for all $\lambda \in(0,1)$. In fact, $0 \in \operatorname{ext}(S)$ cannot hold either. For, given that $A$ is compact, by Milman's converse to the Krein-Milman theorem, we have $\operatorname{ext}(S)=\operatorname{ext}(\overline{\mathrm{co}}(A)) \subseteq A$, so $0 \in \operatorname{ext}(S)$ would imply that $0 \in A$. But this would in turn yield a strictly increasing sequence $\left(n_{k}\right)$ of positive integers such that $\left\|T^{n_{k}} y\right\| \rightarrow 0$ as $k \rightarrow \infty$, which is impossible in view of (3). Thus $0 \notin S$ and the proof is complete.

Whether Theorem 2.1.A would remain valid if $K$ was taken only to be weakly compact is an open problem. However, the above proof modifies easily to show that this is the case at least for Dunford-Pettis operators.

Theorem 2.1.B. Let $X$ be a Banach lattice, and let $T \in \mathcal{L}_{+}(X)$ be a power bounded Dunford-Pettis operator that commutes with a weakly compact operator $K \in \mathcal{K}^{w}(X)$. Then $T$ has a nonzero fixed point in $X_{+}$provided that (3) holds.

Proof. Choosing any $x \in K^{-1}(y)$, in this case we define $A:=\operatorname{cl}^{\mathrm{w}}\left(\mathcal{O}_{T}(y)\right)$ and $S:=\overline{\mathrm{co}}(A)$. By the Krein-Šmulian weak compactness theorem, $S$ is a nonempty convex and weakly compact set in $X$. Moreover, since it is linear, $T$ is weak-to-weak continuous, and hence we may apply the Schauder-Tychonoff fixed point theorem again to find a point of $S$ fixed under $T$. In turn, the argument given in the second paragraph of the proof of Theorem 2.1.A yields a strictly increasing sequence $\left(n_{k}\right)$ of positive integers such that $\mathrm{w}-\lim T^{n_{k}} y=0$. Since $T$ is Dunford-Pettis, this implies that $\lim \left\|T^{n_{k}+1} y\right\|=0$, contradicting (3) . 
The spirit of these results is reminiscent of certain classical results of metric fixed point theory. For instance, a well-known generalization of the famous Kirk fixed point theorem says that every nonexpansive self-map of a weakly compact convex set in a Banach space $X$ has a fixed point, provided that $X$ has weak asymptotic normal structure [1]. Or, it is known that every nonexpansive self-map of a weakly compact convex set in a Banach space with a 1-unconditional basis has a fixed point [12. Theorems 2.1.A-B depart from such results in that they deal with the existence of nonzero fixed points. In addition, owing to their linear nature, they do not require the Banach space under consideration to have a special structure such as having a Schauder basis or normal structure.

The following important special case of Theorems 2.1.A-B may be worth emphasizing.

Corollary 2.1. Let $X$ be a Banach lattice, and let $T \in \mathcal{L}_{+}(X)$ be a power-bounded and power-compact operator. $T$ has a nonzero fixed point in $X_{+}$if and only if $\liminf \left\|T^{n} x\right\|>0$ for some $x \in X_{+}$.

Proof. Pick any positive integer $n$ with $T^{n} \in \mathcal{K}(X)$, define $K:=T^{n}$ and $y:=T^{n} x$, and apply Theorem 2.1.A.

We shall show in Section 2.2 that Corollary 2.1 can be substantially generalized in the case of AL-spaces.

Remark 2.1. Corollary 2.1 is tight in the sense that none of its hypotheses can be dropped in its statement. (A similar remark applies to Theorem 2.2 below.) That this is the case for power boundedness and positivity is shown by trivial examples on $\mathbb{R}$. The weighted right-shift operator $T\left(x_{1}, x_{2}, \ldots\right):=\left(x_{2}, 2^{-1} x_{3}, 3^{-1} x_{4} \ldots\right)$ lacks a nonzero fixed point, but it is a positive compact contraction on $\ell^{2}$. Finally, the operator $T \in \mathcal{B}(C[0,1])$ defined by $T(f)(t):=t f(t)$ is a positive contraction such that $\left\|T^{n}\left(1_{[0,1]}\right)\right\|_{\infty}=\sup _{0 \leq t \leq 1}\left|t^{n}\right|=1$ for each $n$. But it is plain that $T$ does not have a nonzero fixed point.

Remark 2.2. (a) One does not in fact need the full strength of power-boundedness in Corollary 2.1. Indeed, if $T \in \mathcal{L}_{+}(X)$ is power-compact, then it has a positive fixed point iff there exists an $x \in X_{+}$such that

$$
\infty>\limsup _{n \rightarrow \infty}\left\|T^{n} x\right\| \geq \liminf _{n \rightarrow \infty}\left\|T^{n} x\right\|>0 .
$$

The proof is analogous to that of Theorem 2.1.A.

(b) A well-known result of Krein and Rutman 9] says that if $T \in \mathcal{L}_{+}(X)$ is power-compact, then the spectral radius $r(T)$ is an eigenvalue of $T$ corresponding to an eigenvector in $X_{+}$. The Gelfand spectral formula implies that if $T$ is nonexpansive and not strongly stable, then $r(T)=1$, so in this case the said Krein-Rutman theorem guarantees that $T$ has a nonzero fixed point in $X_{+}$. Corollary 2.1 can be viewed as a modest generalization of this observation.

The final result of this subsection illustrates how Corollary 2.1 can be utilized in certain Banach sequence spaces. We first make note of the following version of the discrete Fubini theorem.

Lemma 2.1. Let $(X, \succsim)$ be a Banach lattice with an order-continuous norm. If $\left(x_{m k}\right)$ is a double sequence in $X_{+}$such that $\sum_{k} \sum_{m} x_{m k}$ converges, then $\sum_{m} \sum_{k} x_{m k}$ also converges and $\sum_{m} \sum_{k} x_{m k}=\sum_{k} \sum_{m} x_{m k}$. 
Proof. Define $y:=\sum_{k} \sum_{m} x_{m k}$ and let

$$
y_{n}:=y-\sum_{n \geq m \geq 1} \sum_{k \geq 1} x_{m k}
$$

for any $n \in \mathbb{N}$. Clearly, $y_{1} \succsim y_{2} \succsim \cdots \succsim 0$, so by order-continuity, $\left(y_{n}\right)$ is convergent. This implies that $y \succsim \sum_{m} \sum_{k} x_{m k} \in X_{+}$. Interchanging the roles of $m$ and $k$, we also get $\sum_{m} \sum_{k} x_{m k} \succsim y$, and the result follows.

Corollary 2.2. Let $X$ be a real Banach space with an unconditional Schauder basis $\left(e^{k}\right)$ such that

$$
\sum_{k \geq 1}\left\langle x, e^{k}\right\rangle<\infty \quad \text { for all } x \in X .
$$

Let $T \in \mathcal{B}(X)$ be a power-bounded and power-compact operator that satisfies the following two properties:

(i) $\left\langle T e^{k}, e^{m}\right\rangle \geq 0$ for all $k, m=1,2, \ldots$,

(ii) $\liminf \left\|T^{n} y\right\|>0$ for some $y \in X$ with $\left\langle y, e^{k}\right\rangle \geq 0$ for all $k$.

Then there exists a nonzero $x \in X$ with $x=T x$ and $\left\langle x, e^{k}\right\rangle \geq 0$ for all $k$.

Proof. Let $\succsim$ stand for the coordinatewise partial order on $X$, that is, $z \succsim w$ iff $\left\langle z, e^{k}\right\rangle \geq\left\langle w, e^{k}\right\rangle$ for all $k$. Define the map $\|\cdot\|^{\prime}: X \rightarrow \mathbb{R}_{+}$by $\left\|\sum \alpha_{k} e^{k}\right\|^{\prime}:=$ $\sup \left\{\left\|\sum \beta_{k} \alpha_{k} e^{k}\right\|:\left(\beta_{k}\right) \in S_{\ell \infty}\right\}$, and note that $\left(X, \succsim,\|\cdot\|^{\prime}\right)$ is a Banach lattice (Theorem 4.2.22 of [13]). Now take any $\succsim$-decreasing sequence $\left(z_{m}\right)$ with $\inf _{m \geq 1} z_{m}=0$, and observe that since $\sum\left\langle z_{1}, e^{k}\right\rangle<\infty$, we can apply the monotone convergence theorem to get $\lim _{m} \sum_{k}\left\langle z_{m}, e^{k}\right\rangle=\sum_{k} \lim _{m}\left\langle z_{m}, e^{k}\right\rangle=0$. But $\left\|z_{m}\right\|^{\prime} \leq \sum_{k}\left\langle z_{m}, e^{k}\right\rangle$ for each $m$, so we have $\left\|z_{m}\right\|^{\prime} \searrow 0$ as $m \rightarrow \infty$. Since it is easily seen that $(X, \succsim)$ is Dedekind complete, it follows that $\left(X, \succsim,\|\cdot\|^{\prime}\right)$ is a Banach lattice with an ordercontinuous norm.

Now fix an arbitrary $z$ with $\left\langle z, e^{m}\right\rangle \geq 0$ for all $m$, and note that

$$
T z=\sum_{k \geq 1} \sum_{m \geq 1}\left\langle z, e^{k}\right\rangle\left\langle T e^{k}, e^{m}\right\rangle e^{m}=\sum_{m \geq 1} \sum_{k \geq 1}\left\langle z, e^{k}\right\rangle\left\langle T e^{k}, e^{m}\right\rangle e^{m}
$$

where the second equality follows from Lemma 2.1. Then, by (i),

$$
\left\langle T z, e^{m}\right\rangle=\sum_{k \geq 1}\left\langle z, e^{k}\right\rangle\left\langle T e^{k}, e^{m}\right\rangle \geq 0, \quad m=1,2, \ldots .
$$

Thus $T \in \mathcal{L}_{+}(X)$, and we can apply Corollary 2.1 to complete the proof.

2.2. The case of quasicompact operators. This subsection is concerned with quasicompact positive operators defined on a Banach lattice. The main result provides a sufficient condition for the existence of a positive fixed point in terms of the sequence of Cesaro means associated with the iterations of a positive vector. This result is very much in the same spirit with the famous Yosida-Kakutani mean ergodic theorem, and it is indeed proved by means of a similar method. As in the previous subsection, the main difference here is that the present approach allows one to obtain a nonzero fixed point for the operator in question 1

\footnotetext{
${ }^{1}$ Particularly related to Theorem 2.2 is Theorem 3 of [18], a special case of which shows that every weakly quasicompact operator in a Banach space has a fixed point. However, Yosida and Kakutani prove this result by using their mean ergodic theorem; the present proof is more direct.
} 
Theorem 2.2. Let $X$ be a Banach lattice, and let $T \in \mathcal{L}_{+}(X)$ be a power-bounded quasicompact operator. $T$ has a nonzero fixed point in $X_{+}$if and only if

$$
\liminf _{m \rightarrow \infty}\left\|\frac{1}{m} \sum_{i=1}^{m} T^{i} y\right\|>0 \quad \text { for some } y \in X_{+} .
$$

Proof. Pick any positive integer $n$ with $\left\|T^{n}-K\right\|<1$ for some $K \in \mathcal{K}(X)$, and define $L:=T^{n}-K$. Since $\|L\|<1, I-L$ is invertible and $(I-L)^{-1}=\sum_{i \geq 0} L^{i} \in$ $\mathcal{B}(X)$. Define

$$
U_{m}:=\frac{1}{m} \sum_{i=1}^{m} T^{i}, \quad m=1,2, \ldots,
$$

and observe that each $U_{m}$ is a positive operator on $X$ with $\left\|U_{m}\right\| \leq \sup _{i \geq 1}\left\|T^{i}\right\|=$ : $s(T)$. Moreover, for each $m$,

$$
\begin{aligned}
U_{m} & =(I-L)^{-1}(I-L) U_{m} \\
& =(I-L)^{-1}\left(I-T^{n}+K\right) U_{m} \\
& =(I-L)^{-1}\left(I-T^{n}\right) U_{m}+\left((I-L)^{-1} K\right) U_{m} .
\end{aligned}
$$

Since $(I-L)^{-1} K \in \mathcal{K}(X)$ and since $\left\{U_{m} y: m \in \mathbb{N}\right\}$ is bounded, the set $(I-L)^{-1} K\left(\left\{U_{m} y: m \in \mathbb{N}\right\}\right)$ is relatively compact. So, there exist a strictly increasing sequence $\left(m_{k}\right)$ in $\mathbb{N}$ and a $z \in X$ such that

$$
\lim _{k \rightarrow \infty}(I-L)^{-1} K U_{m_{k}} y=z
$$

On the other hand, for $m>n$ it is readily verified that

$$
\begin{aligned}
\left\|\left(I-T^{n}\right) U_{m}\right\| & =\frac{1}{m}\left\|T+\cdots+T^{m}-T^{n+1}-\cdots-T^{n+m}\right\| \\
& =\frac{1}{m}\left\|T+\cdots+T^{n}-T^{m+1}-\cdots-T^{m+n}\right\| \\
& =\frac{1}{m}\left\|\left(\sum_{i=1}^{n} T^{i}\right)\left(I-T^{m}\right)\right\| \\
& \leq \frac{1}{m} n s(T)(1+s(T)) .
\end{aligned}
$$

Therefore, $\left(I-T^{n}\right) U_{m} \rightarrow 0$ as $m \rightarrow \infty$. Combining this finding with (55) and (6), we get $\lim U_{m_{k}} y=z$ so that $\lim T U_{m_{k}} y=T z$. But

$$
\left\|T U_{m_{k}} y-U_{m_{k}} y\right\|=\frac{1}{m_{k}}\left\|T^{m_{k}} y-y\right\| \leq \frac{1}{m_{k}}(s(T)+\|y\|)
$$

so that $\left\|T U_{m_{k}} y-U_{m_{k}} y\right\| \rightarrow 0$ as $k \rightarrow \infty$. Thus, we must have $T z=z$. That $z \in X_{++}$is an obvious consequence of the positivity of $T$ and (4).

The following result generalizes Corollary 2.1 for operators defined on an ALspace, that is, on a Banach lattice the norm of which is additive on its positive cone.

Corollary 2.3. Let $X$ be an $A L$-space, and let $T \in \mathcal{L}_{+}(X)$ be a power-bounded quasicompact operator. $T$ has a nonzero fixed point in $X_{+}$if and only if $\lim \inf \left\|T^{n} y\right\|>$ 0 for some $y \in X_{+}$. 
Proof. We only need to verify (4). Since $\liminf \left\|T^{n} y\right\|>0$, there exists an $\alpha>0$ such that $\left\|T^{n} y\right\| \geq \alpha$ for all $n$. But $T^{n} y \in X_{+}$for each $n$, so by additivity of $\|\cdot\|$ on $X_{+}$, we get $\left\|m^{-1} \sum_{i=0}^{m-1} T^{i} y\right\|=m^{-1} \sum_{i=0}^{m-1}\left\|T^{i} y\right\| \geq \alpha$ for each $m$.

Remark 2.3. For Dunford-Pettis operators, quasicompactness can be replaced with positive weak quasicompactness in the statements of Theorem 2.2 and Corollary 2.3. To prove this, we adopt the notation given in the proof of Theorem 2.2, and assume that $K$ is weakly compact and positive, and $T^{n}-K$ is positive. We have $U_{m}=V_{m}+W_{m}$ where $V_{m}:=(I-L)^{-1}\left(I-T^{n}\right) U_{m}$ and $W_{m}:=(I-L)^{-1} K U_{m}$. Clearly, $V_{m} \rightarrow 0$ as $m \rightarrow \infty$ and $W_{m} \in \mathcal{L}_{+}(X)$ for each $m$. The arguments above (along with the Eberlein-Šmulian theorem) yield a strictly increasing sequence $\left(m_{k}\right)$ in $\mathbb{N}$ and a $z \in X$ such that w- $\lim U_{m_{k}} y={ }_{\mathrm{w}}-\lim W_{m_{k}} y=z=T z$. We need to show that $z \in X_{++}$. To this end, define $O:=\left\{W_{m} y: m \in \mathbb{N}\right\}$ and $A:=\mathrm{cl}^{\mathrm{w}}(O)$. Since $\sup _{m \geq 1}\left\|U_{m}\right\| \leq s(T)$ and $(I-L)^{-1} K$ is weakly compact, $A$ is a weakly compact set in $X_{+}$with $z \in A$. It is thus enough to show that $0 \notin S:=\overline{\mathrm{co}}(A)$ to prove the claim. But if $0 \in S$, by using the argument given in the second paragraph of the proof of Theorem 2.1.A, we may obtain a strictly increasing sequence $\left(m_{t}\right)$ in $\mathbb{N}$ with w-lim $W_{m_{t}} y=0$. Since each $W_{m}$ is Dunford-Pettis, this gives $\left\|W_{m_{t}} y\right\| \rightarrow 0$, which in turn yields $\left\|U_{m_{t}} y\right\| \leq\left\|V_{m_{t}} y\right\|+\left\|W_{m_{t}} y\right\| \rightarrow 0$ as $t \rightarrow \infty$, contradicting (4).

\section{Application: On the existence of invariant Densities FOR MARKOV OPERATORS}

Let $(S, \Sigma, \mu)$ be an arbitrary $\sigma$-finite measure space. We adopt the convention of denoting the real Banach space $L^{1}(S, \Sigma, \mu)$ by $L^{1}$. When ordered pointwise (so that $L_{+}^{1}=\left\{f \in L^{1}: f \geq 0\right.$ a.e. $\}$ ) this space is a Banach lattice. A linear operator $T$ on $L^{1}$ is called a Markov operator if it is a positive contraction which is norm-preserving on $L_{+}^{1}$, that is, if $\|T\|_{1} \leq 1$, and $T f \geq 0$ a.e. and $\|T f\|_{1}=\|f\|_{1}$ hold whenever $f \geq 0$ a.e. We denote the set of all Markov operators on $L^{1}$ by $\mathcal{M}(S, \Sigma, \mu)$. A function $f$ in $L^{1}$ is called a density if $f \in L_{+}^{1}$ and $\|f\|_{1}=1$. The set of all densities is denoted as $D$. By definition, $D$ is invariant under any Markov operator $T$, that is, $T(D) \subseteq D$. If $D \ni f=T f$, then $f$ is called an invariant density for $T$.

Proposition 3.1. Let $(S, \Sigma, \mu)$ be a $\sigma$-finite measure space, and $T \in \mathcal{M}(S, \Sigma, \mu)$. If $T$ commutes with a nonzero compact operator $K$ on $L^{1}(S, \Sigma, \mu)$, then there exists an invariant density for $T$.

Proof. Take any $g \in L^{1}$ with $K g \neq 0$ a.e., and define the map $h: S \rightarrow \mathbb{R}$ by

$$
h(s):=\left\{\begin{aligned}
g(s), & \text { if } K g(s) \geq 0, \\
-g(s), & \text { if } K g(s)<0 .
\end{aligned}\right.
$$

It is obvious that $h \in L^{1}$ and that $L_{+}^{1} \ni K h \neq 0$ a.e. Thus letting $f:=$ $K\left(h /\|K h\|_{1}\right)$, we find that $f \in D \cap K\left(L^{1}\right)$, and $\left\|T^{n} f\right\|_{1}=\|f\|_{1}>0$ for all $n=1,2, \ldots$. The claim then follows from Theorem 2.1.A.

As we shall show in terms of Markov chains below (see Proposition 3.3), one advantage of this result is that the sufficient condition that it gives for the existence of an invariant density for a Markov operator is not in terms of the asymptotic behavior of the sequence of iterations of the operator. This contrasts with most of the related results that appear in the literature, for instance, with those given in [4], [11], [16], and [7]. 
Our next application is a common invariant density theorem which follows almost immediately from Corollary 2.3.

Proposition 3.2. Let $(S, \Sigma, \mu)$ be a $\sigma$-finite measure space. If $\mathcal{T}$ is a finite commuting family of quasicompact operators in $\mathcal{M}(S, \Sigma, \mu)$, then there exists an invariant density common to all members of $\mathcal{T}$.

Proof. Define $\Psi(T):=\left\{f \in L^{1}: f=T f\right\}$ for each $T \in \mathcal{T}$. By Corollary 2.3, $\Psi(T)$ is a closed subspace of $L^{1}$ such that $\Psi(T) \cap D \neq \emptyset$ for all $T \in \mathcal{T}$. For any $T_{1}$ and $T_{2}$ in $\mathcal{T}$, we have $T_{2}\left(\Psi\left(T_{1}\right)\right) \subseteq \Psi\left(T_{1}\right)$ since $T_{1} T_{2}=T_{2} T_{1}$. Thus $\left.T_{2}\right|_{\Psi\left(T_{1}\right)}$ is a quasicompact contraction on the AL-space $\Psi\left(T_{1}\right)$, and, for any $f \in \Psi\left(T_{1}\right) \cap D \neq \emptyset$, we have $\left\|T_{2}^{n} f\right\|_{1}=\|f\|_{1}>0$ for all $n$. Thus Corollary 2.3 applies, and we find that $\Psi\left(T_{1}\right) \cap \Psi\left(T_{2}\right) \cap D \neq \emptyset$. But if $T_{3}$ is any other member of $\mathcal{T}$, then $T_{3}\left(\Psi\left(T_{1}\right) \cap \Psi\left(T_{2}\right)\right) \subseteq$ $\Psi\left(T_{1}\right) \cap \Psi\left(T_{2}\right)$, so continuing inductively, we find $\cap\{\Psi(T): T \in \mathcal{T}\} \cap D \neq \emptyset$.

Finally, we illustrate the applicability of these results by examining their immediate implications for the theory of Markov chains. Let $\mathbb{R}^{\infty \times \infty}$ denote the class of all infinite real matrices and recall that a matrix $P=\left[p_{i j}\right]_{\mathbb{N} \times \mathbb{N}} \in \mathbb{R}^{\infty \times \infty}$ is said to be stochastic if $p_{i j} \geq 0$ and $\sum_{j} p_{i j}=1$ for all $i, j \in \mathbb{N}$. Letting $\ell^{1}:=L^{1}\left(\mathbb{N}, 2^{\mathbb{N}}, \mu_{c}\right)$, where $\mu_{c}$ is the counting measure, $P$ is viewed as a positive linear operator on $\ell^{1}$ in the obvious way: $P:\left(x_{1}, x_{2}, \ldots\right) \mapsto\left(\sum_{i \geq 1} p_{i 1} x_{i}, \sum_{i \geq 1} p_{i 2} x_{i}, \ldots\right)$. Since

$$
\|P x\|_{1}=\sum_{j \geq 1} \sum_{i \geq 1} p_{i j} x_{i}=\sum_{i \geq 1} x_{i} \sum_{j \geq 1} p_{i j}=\sum_{i \geq 1} x_{i}=\|x\|_{1}
$$

for any $x \equiv\left(x_{1}, x_{2}, \ldots\right) \in \ell_{+}^{1}, P$ is a Markov operator on $\ell^{1}$. It is also easy to show that any Markov operator on $\ell^{1}$ arises this way, so we may identify the set of all stochastic matrices in $\mathbb{R}^{\infty \times \infty}$ with $\mathcal{M}:=\mathcal{M}\left(\mathbb{N}, 2^{\mathbb{N}}, \mu_{c}\right)$. Of course, in probability theory, a member of $\mathcal{M}$ is referred to as a homogeneous Markov chain with a countably infinite state space, and an invariant density for it is called a stationary distribution of the chain.

Lemma 3.1. If $P \in \mathcal{M}$ and

$$
\lim _{k \rightarrow \infty} \sup _{i \geq 1} \sum_{j \geq k+1} p_{i j}=0,
$$

then $P$ is a compact operator on $\ell^{1} 2$

Proof. For any $k \in \mathbb{N}$, define $P_{k} \in \mathcal{L}\left(\ell^{1}\right)$ by

$$
P_{k} x:=\left(\sum_{i=1}^{k} p_{i 1} x_{i}, \ldots, \sum_{i=1}^{k} p_{i k} x_{i}, 0,0, \ldots\right) .
$$

Then, for any $x \in S_{\ell^{1}}$,

$$
\left\|\left(P-P_{k}\right) x\right\|_{1} \leq \sum_{i \geq 1}\left|x_{i}\right| \sum_{j \geq k+1} p_{i j} \leq \sum_{i \geq 1}\left|x_{i}\right|\left(\sup _{i \geq 1} \sum_{j \geq k+1} p_{i j}\right)=\sup _{i \geq 1} \sum_{j \geq k+1} p_{i j},
$$

so, by hypothesis, $\left\|P-P_{k}\right\|_{1} \rightarrow 0$ as $k \rightarrow \infty$. Since each $P_{k}$ is of finite rank, $P$ must be compact.

\footnotetext{
${ }^{2}$ One can also show that (7) is in fact necessary for the compactness of $P \in \mathcal{M}$, but we do not need to prove this here. See, for instance, Example 3 of [17], p. 278.
} 
Proposition 3.3. Let $P, Q \in \mathcal{M}$ and $0 \leq \beta \leq 1$. If (77) holds, and if $\beta Q$ is algebraic, then $\beta Q+(1-\beta) P \in \mathcal{M}$ has a stationary distribution.

Proof. Let $U:=\beta Q$ and $V:=(1-\beta) P$. Since $U$ is algebraic, there exist an $n \in \mathbb{N}$ and $\alpha_{0}, \ldots, \alpha_{n} \in \mathbb{Z}$ such that $\sum_{i=1}^{n} \alpha_{i} U^{i}=0$. Writing $p(T)$ for $\sum_{i=1}^{n} \alpha_{i} T^{i}$ for any $T \in \mathcal{B}\left(\ell^{1}\right)$, we have

$$
p(U+V)=\sum_{i=1}^{n} \alpha_{i} U^{i}+\sum_{i=1}^{n} \alpha_{i} \sum_{r=0}^{i-1}\left(\begin{array}{l}
i \\
r
\end{array}\right) U^{r} V^{i-r}=\sum_{i=1}^{n} \alpha_{i} \sum_{r=0}^{i-1}\left(\begin{array}{l}
i \\
r
\end{array}\right) U^{r} V^{i-r} .
$$

But $V$ is compact by Lemma 3.1, so this computation shows that $p(U+V) \in$ $\mathcal{K}\left(\ell^{1}\right)$. Since $U+V$ obviously commutes with $p(U+V)$, it must have a stationary distribution by Proposition 3.1.

Similarly, this time using Proposition 3.2, one can show that any $P \in \mathcal{M}$, for which there is some $Q \in \mathcal{K}\left(\ell^{1}\right)$ with $\sup _{i \geq 1} \sum_{j \geq k+1}\left|p_{i j}-q_{i j}\right| \rightarrow 0$ as $k \rightarrow \infty$, has a stationary distribution.

\section{NONZERO FIXED POINTS OF UNIFORMLY ASYMPTOTICALLY REGULAR CONTRACTIONS}

All of the fixed point theorems reported in Section 2 were based on an operatorcompactness property that forbids the orbits of an operator to grow "too large." Even in the absence of such a compactness property, however, one may ensure the existence of a nonzero fixed point by verifying that these orbits are in fact well-behaved. In metric fixed point theory, a nontrivial property that is frequently used for this purpose is asymptotic regularity which was introduced originally by Browder and Petryshyn [3]. We shall show below that, in the case of Hilbert spaces, the uniform version of this property does the work of both positivity and compactness (or quasicompactness, etc.) insofar as the nonzero fixed points of a linear operator is concerned.

Definition. Let $X$ be a normed linear space and $T \in \mathcal{B}(X)$. We say that $T$ is asymptotically regular if

$$
\lim _{n \rightarrow \infty}\left\|T^{n} x-T^{n+1} x\right\|=0 \quad \text { for all } x \in X .
$$

If the convergence of this limit is uniform, that is, $\left\|T^{n}-T^{n+1}\right\| \rightarrow 0$ as $n \rightarrow \infty$, we then say that $T$ is uniformly asymptotically regular.

Uniformly asymptotically regular and power-bounded operators enjoy interesting spectral properties. In particular, a power-bounded operator whose spectrum crosses the unit circle admits 1 in its spectrum iff it is uniformly asymptotically regular (cf. [8]). The following lemma, on the other hand, shows that a uniformly asymptotically regular and power-bounded operator admits 1 as an eigenvalue iff it is not strongly stable.

Lemma 4.1. Let $X$ be a complex Hilbert space and let $T \in \mathcal{B}(X)$ be power-bounded and uniformly asymptotically regular. Then $T$ has a nonzero fixed point if and only if it is not strongly stable.

Proof. We only need to establish the sufficiency part of the claim. To this end, we begin by observing that, by power-boundedness, $\|\cdot\|^{\prime}: X \rightarrow \mathbb{R}_{+}$defined by $\|x\|^{\prime}:=$ $\sup _{n \geq 1}\left\|T^{n} x\right\|$ is a norm on $X$ which is equivalent to $\|\cdot\|$ and with respect to which 
$T$ is nonexpansive. Therefore, it is enough to prove the lemma for nonexpansive $T$, so we assume in what follows that $\|T\| \leq 1$.

Since $T$ is not strongly stable, we can find a $y \in S_{X}$ with $\lim \|T\|^{n} \geq \lim \left\|T^{n} y\right\|=$ : $\alpha>0$ which would not be possible if $\|T\|<1$. Thus $\|T\|=1$. On the other hand, since $\left(\left\|T^{n} y\right\|\right)$ is a decreasing sequence, $\left\|T^{n}\right\| \geq\left\|T^{n} y\right\| \geq \alpha$ for each $n$, and hence, by the Gelfand spectral radius formula, $r(T)=\lim \left\|T^{n}\right\|^{1 / n} \geq \lim \alpha^{1 / n}=1$. Consequently, we have $1=\|T\| \geq r(T) \geq 1$, that is, $r(T)=1$. Since $\sigma(T)$ is compact, it follows that there exists a $\lambda \in \mathbb{C}$ with $|\lambda|=1$ and $\lambda \in \sigma(T)$. But if $\lambda \neq 1$, then the spectral mapping theorem yields

$$
\left\|T^{n}-T^{n+1}\right\| \geq r\left(T^{n}-T^{n+1}\right) \geq|1-\lambda|, \quad n=1,2, \ldots,
$$

which is impossible due to the uniform asymptotic regularity of $T$. Thus we conclude that $1 \in \sigma(T)=\sigma_{p}(T) \cup \sigma_{c}(T) \cup \sigma_{r}(T)$. To complete the proof, then, it is enough to show that $1 \notin \sigma_{c}(T) \cup \sigma_{r}(T)$.

If $1 \in \sigma_{r}(T)$, then because $\sigma_{r}(T) \subseteq\left\{\bar{\lambda}: \lambda \in \sigma_{p}\left(T^{*}\right)\right\}$, we find $1 \in \sigma_{p}\left(T^{*}\right)$, that is, $T^{*}(x)=x$ (so that $\left\langle x, T^{*} x\right\rangle=\|x\|^{2}$ ) for some $x \in S_{X}$. But $1 \in \sigma_{r}(T)$ implies $\operatorname{ker}(I-T)=\{0\}$, so we have $x \neq T x$. Combining these two observations, we get

$$
0<\|T x-x\|^{2}=\|T x\|^{2}+\|x\|^{2}-2 \operatorname{Re}\left\langle x, T^{*} x\right\rangle=\|T x\|^{2}-1,
$$

contradicting $\|T\|=1$.

Assume next that $1 \in \sigma_{c}(T)$ so that $\operatorname{cl}((I-T)(X))=X$. Then there exists a sequence $x_{k}$ in $X$ such that $x_{k}-T x_{k} \rightarrow y$ as $k \rightarrow \infty$. So, for each $\varepsilon>0$, there exists a $k_{0}>0$ such that, for every $n \in \mathbb{N}$,

$$
\left\|T^{n} x_{k}-T^{n+1} x_{k}-T^{n} y\right\|=\left\|T^{n}\left(x_{k}-T x_{k}-y\right)\right\| \leq\left\|x_{k}-T x_{k}-y\right\|<\varepsilon
$$

whenever $k \geq k_{0}$. Therefore,

$$
\left\|T^{n} x_{k}-T^{n+1} x_{k}-T^{n} y\right\| \rightarrow 0 \text { uniformly as } k \rightarrow \infty .
$$

Moreover, $\left(\left\|T^{n}\left(x_{k}-T x_{k}-y\right)\right\|\right)$ converges as $n \rightarrow \infty$ since $T$ is a contraction. Therefore, by using the asymptotic regularity of $T$,

$$
\lim _{n \rightarrow \infty}\left\|T^{n} x_{k}-T^{n+1} x_{k}-T^{n} y\right\| \geq \lim _{n \rightarrow \infty}\left|\left\|T^{n} x_{k}-T^{n+1} x_{k}\right\|-\left\|T^{n} y\right\|\right|=\alpha .
$$

So, by (8) and the Moore-Osgood theorem, we get

$$
\alpha \leq \lim _{k \rightarrow \infty} \lim _{n \rightarrow \infty}\left\|T^{n} x_{k}-T^{n+1} x_{k}-T^{n} y\right\|=\lim _{n \rightarrow \infty} \lim _{k \rightarrow \infty}\left\|T^{n} x_{k}-T^{n+1} x_{k}-T^{n} y\right\|=0 .
$$

This contradiction completes the proof.

We next extend Lemma 4.1 to the case of a real Hilbert space $X$. The complexifcation of $X$, denoted $X_{\mathbb{C}}$, is the additive group $X \oplus i X$ with the scalar multiplication $(a+i b)(x+i y):=(a x-b y)+i(a y+b x)$. There are numerous ways of norming $X_{\mathbb{C}}$ in such a way that $X$ would be embedded in $X_{\mathbb{C}}$ (cf. [14). A particularly convenient way of doing this for our purposes is through endowing $X_{\mathbb{C}}$ with a suitable inner product. To this end, we define the map $\langle\cdot, \cdot\rangle_{*}: X_{\mathbb{C}} \times X_{\mathbb{C}} \rightarrow \mathbb{C}$ by

$$
\langle x+i y, z+i w\rangle_{*}=\langle x, z\rangle+\langle y, w\rangle+i(\langle y, z\rangle-\langle x, w\rangle)
$$

and note that $\left(X_{\mathbb{C}},\langle\cdot, \cdot\rangle_{*}\right)$ is a complex Hilbert space in which $X$ is naturally embedded. Moreover, if $\|\cdot\|_{*}$ stands for the norm induced by $\langle\cdot, \cdot\rangle_{*}$, then we have the identity $\|x+i y\|_{*}^{2} \equiv\|x\|^{2}+\|y\|^{2}$. Finally, notice that every (real-)linear operator $T$ on $X$ uniquely extends to a (complex-)linear operator $T_{\mathbb{C}}$ on $X_{\mathbb{C}}$ through the formula $T_{\mathbb{C}}(x+i y)=T x+i T y$. We denote the operator norm on $\mathcal{B}\left(X_{\mathbb{C}}\right)$ by 
$\|\cdot\|_{*}$. Obviously, $\mathcal{B}(X)$ can be identified with a real subspace of $\mathcal{B}\left(X_{\mathbb{C}}\right)$. Moreover, if $T \in \mathcal{B}(X)$, then, since $T_{\mathbb{C}}$ extends $T$, we have $\left\|T_{\mathbb{C}}\right\|_{*} \geq\|T\|$. Conversely, for any $x, y \in X$, we have

$$
\begin{aligned}
\|T\|^{2}\|x+i y\|_{*}^{2} & =(\|T\|\|x\|)^{2}+(\|T\|\|y\|)^{2} \geq\|T x\|^{2}+\|T y\|^{2}=\|T x+i T y\|_{*}^{2} \\
& =\left\|T_{\mathbb{C}}(x+i y)\right\|_{*}^{2}
\end{aligned}
$$

so that $\left\|T_{\mathbb{C}}\right\|_{*} \leq\|T\|$. That is, $\left\|T_{\mathbb{C}}\right\|_{*}=\|T\|$ for any $T \in \mathcal{B}(X)$.

With this preparation, the main result of this section is easily proved.

Theorem 4.1. Let $X$ be a real Hilbert space and let $T \in \mathcal{B}(X)$ be power-bounded and uniformly asymptotically regular. Then $T$ has a nonzero fixed point if and only if it is not strongly stable.

Proof. By induction, $\left(T^{n}\right)_{\mathbb{C}}=T_{\mathbb{C}}^{n}$ and $\left(T^{n}-T^{n+1}\right)_{\mathbb{C}}=T_{\mathbb{C}}^{n}-T_{\mathbb{C}}^{n+1}$, so $\left\|T_{\mathbb{C}}^{n}\right\|_{*}=$ $\left\|T^{n}\right\|$ and $\left\|T_{\mathbb{C}}^{n}-T_{\mathbb{C}}^{n+1}\right\|_{*}=\left\|T^{n}-T^{n+1}\right\|$ for each $n \in \mathbb{N}$. Thus $T_{\mathbb{C}} \in \mathcal{B}\left(X_{\mathbb{C}}\right)$ is power-bounded and uniformly asymptotically regular. But it is obvious that $T_{\mathbb{C}}$ is not strongly stable, and hence, by Lemma 4.1, there exist $x, y \in X$ such that $T_{\mathbb{C}}(x+i y)=x+i y$ where either $x$ or $y$ is not zero. Any nonzero member of $\{x, y\}$ is a fixed point of $T$.

Remark 4.1. The postulated inner product structure is essential for the validity of Theorem 4.1. For instance, let $X:=C[0,1]$ and consider the contraction $T \in \mathcal{B}(X)$ defined by $T(f)(t):=t f(t)$ for each $t \in[0,1]$. While $T$ lacks a nonzero fixed point and is not strongly stable, it is uniformly asymptotically regular, for

$$
\begin{aligned}
\left\|T^{n}-T^{n+1}\right\|_{\infty} & =\sup _{f \in S_{X}} \sup _{0 \leq t \leq 1}\left|t^{n} f(t)-t^{n+1} f(t)\right| \\
& \leq \sup _{0 \leq t \leq 1}\left|t^{n}-t^{n+1}\right| \rightarrow 0 \quad \text { as } n \rightarrow \infty .
\end{aligned}
$$

We conclude by noting that it is not known if the qualifier "uniformly" can be omitted in the statement of Theorem 4.1.

\section{REFERENCES}

[1] Baillon, J. B. and R. Schöneberg (1981), "Asymptotic normal structure and fixed points of nonexpansive mappings," Proc. Amer. Math. Soc., 81, 257-264. MR 82c:47068

[2] Beauzamy, B. (1988), Introduction to Operator Theory and Invariant Subspaces, NorthHolland, Amsterdam. MR 90d:47001

[3] Browder, F. and W. Petryshyn (1966), "The solution by iteration of nonlinear functional equations in Banach spaces," Bull. Amer. Math. Soc., 72, 571-575. MR 32:8155b

[4] Edelstein, M. (1964), "On nonexpansive mappings of Banach spaces," Proc. Camb. Phil. Soc., 60, 439-447. MR 29:1521

[5] Foguel, S. R. (1964), "A counterexample to a problem of Sz.-Nagy," Proc. Amer. Math. Soc., 15, 788-790. MR 29:2646

[6] Goebel, K. and W. A. Kirk (1991), Topics in Metric Fixed Point Theory, Cambridge University Press., Cambridge. MR 92c:47070

[7] Hernández-Lerma, O. and J. B. Lasserre (1998), "Existence and uniqueness of fixed points for Markov operators and Markov processes," Proc. London Math. Soc, 76, 711-736. MR 99f:60119

[8] Katznelson, Y. and L. Tzafriri (1986), "On power bounded operators," J. Funct. Anal., 68, 313-328. MR 88e:47006

[9] Krein, M. G. and M. A. Rutman (1950), "Linear operators leaving invariant a cone in a Banach space," Amer. Math. Soc. Translation, no. 126. MR 12:341b

[10] Kubrusly, C. S. (1997), An Introduction to Models and Decompositions in Operator Theory, Birkhäuser, Boston. MR 98g:47004 
[11] Lasota, A. (1980), "A fixed point theorem and its application in ergodic theory," Tohôku Math. J., 48, 51-56. MR 82c:47071

[12] Lin, P. K. (1985), "Unconditional bases and fixed points of nonexpansive mappings," Pacific J. Math., 116, 69-76. MR 86c:47075

[13] Megginson, R. E. (1998), An Introduction to Banach Space Theory, Springer, New York. MR 99k:46002

[14] Muñoz, G. A., Y. Sarantopoulos and A. Tonge (1999), "Complexifications of real Banach spaces, polynomials and multilinear maps," Studia Math., 134, 1-33. MR 2000g:46009]

[15] Nagy, Sz. and C. Foiaş (1970), Harmonic Analysis of Operators on Hilbert Space, NorthHolland, Amsterdam. MR 43:947

[16] Socala, J. (1988), "On the existence of invariant densities for Markov operators," Ann. Polon. Math., 48, 51-56. MR 89g:28026

[17] Taylor, A. (1958), Introduction to Functional Analysis, John Wiley, New York. MR 20:5411

[18] Yosida, K. and S. Kakutani (1941), "Operator-theoretical treatment of Markoff's process and mean ergodic theorem," Ann. Math., 42, 188-228. MR 2:230e

Department of Economics, New York University, 269 Mercer St., New York, New YORK 10003

E-mail address: efe.ok@nyu.edu 